ISSN 1112-9867

Available online at http://www.jfas.info

\title{
CHARACTERIZATION QUALITATIVE AND POTENTIALITIES OF UTILIZATION OF METHACOMPOSTS OF POULTRY IN THE NURSERIES ABOVEGROUND
}

\author{
Y. M'Sadak* et A. Ben M'Barek
}

Département du Génie des Systèmes Horticoles et du Milieu Naturel. Université de Sousse. Institut Supérieur Agronomique de Chott Mariem, BP 47, 4042 Chott Mariem, Tunisie

Received: 13 May 2016 / Accepted: 19 August 2016 / Published online: 01 September 2016

\begin{abstract}
The Agricultural employment of digestates from biomethanation can be used as an alternative to peat. The solid digestate poultry (called Poultry Methacompost: PMC) can be used as a partial substitute in the manufacture of culture substrates. The humified Organic Matter (OM) of mature substrates can be adopted only with the presence of necessary conditions for the development of the plant especially: respect of $\mathrm{pH}$, percentage favorable of aeration, good sanitation, balanced contents of minerals. The present work aims to evaluate three different PMC from an industrial biomethaniser; one is obtained from the digester and the other two from the decanter (during two different periods). It appears from the physico-chemical characterization undertaken the following key results: In general, the PMC has a high bulk density, insufficient porosity of aeration, while the porosity of retention largely meets the standard. The phosphor and potassium are also eligible. The warranty of quality of PMC ensures greater acceptability and facilitates the incorporation with peat for soilless horticultural plants.
\end{abstract}

Keywords: Poultry manure; Industrial biomethaniser; Poultry Methacompost; Physico-chemical characterization; Nursery aboveground.

Author Correspondence, e-mail: msadak.youssef@yahoo.fr

doi: http://dx.doi.org/10.4314/jfas.v8i3.12 


\section{INTRODUCTION}

Dans une vision intégrée d'une meilleure exploitation des déjections animales générées, la production combinée d'énergie (sous forme de biogaz) et d'un digestat (apte à être utilisé en tant qu'ingrédient des substrats de culture destinés pour la production hors sol des plants) s'avère un enjeu économique et écologique intéressant.

La production agricole de biogaz s'est étendue, tout d'abord, en ayant recours à la valorisation biologique anaérobie du fumier de ferme. Le fumier fût utilisé ainsi dans les installations de biogaz, du fait des grosses quantités disponibles, de sa grande teneur en matière sèche et de la tendance de formation de gaz [1]. Les majeures ressources énergétiques ordinaires comme le charbon, le pétrole et le gaz naturel sont en voie d'épuisement, et il est évalué que la disparition des ressources pétrolières pourrait être dans une cinquantaine d'années [2]. Le bois et d'autres formes de biomasse sont parmi les principales ressources énergétiques renouvelables disponibles [3, 4]. La biomasse, importante source d'énergie durable, représente l'unique source des combustibles [5]. Parmi les ressources énergétiques renouvelables, l'énergie de biomasse est la seule issue des ressources organiques [6]. Cette solution innovante de gestion efficace des matières organiques $[7,8,9,10,11,12]$ constitue une source renouvelable d'énergie propre (appelée encore énergie verte) $[13,9,14,15,16,17,18,19,12]$. Il s'agit d'un processus naturel de biodégradation organique, réalisé par l'action concertée d'un consortium microbien complexe [20].

Le traitement des ressources organiques devient d'une grande nécessité à l'échelle tant locale que planétaire [9]. Les ressources en biomasse animale sont généralement attirantes à la transformation quand elles sont engendrées en quantités importantes et régulières [21]. Leur bioconversion anaérobie ou Biométhanisation est considérée comme une solution intéressante sur les plans environnemental (Réduction de la charge polluante), énergétique (Production de biogaz) et agronomique (Emploi agricole direct et/ou indirect des effluents). En Tunisie, il existe principalement deux grandes filières de Biométhanisation d'après le type de biomasse traitée : la valorisation des effluents solides humides générés par les activités agroalimentaire et agricole, et la valorisation des effluents liquides provenant des stations d'épuration des eaux usées [22].

La Biométhanisation est un bioprocédé de traitement des résidus organiques solides et liquides [23], qui amène à la formation d'un produit principal appelé biogaz, composé essentiellement de dioxyde de carbone et de Biométhane et d'un produit résiduaire liquide, appelé digestat [9]. Le 
digestat (Co-produit secondaire) peut être valorisé de deux façons : soit intégré comme matière première dans une chaîne de compostage de déchets organiques solides, soit épandu directement sur les sols agricoles comme amendement. Le post-traitement du digestat conduit généralement à l'obtention d'un digestat solide (dénommé méthacompost) et d'un digestat liquide (appelé jus de process ou lixiviat) $[9,24]$. L'exploitation de ce résidu de la Biométhanisation, est indispensable pour améliorer cette voie de traitement anaérobie, afin de ne pas générer un nouveau type de souillure [25].

La digestion anaérobie est un mode conservatif pour les éléments n'entrant pas dans la composition gazeuse. Ce mode permet donc la préservation des éléments minéraux majeurs (azote, phosphore et potassium), et des oligo-éléments dans le digestat brut [26]. Par conséquent, il y a conservation des éléments fertilisants, mais également des polluants potentiels. Pour ce qui est des qualités fertilisantes au sens strict, les changements essentiels qui s'opèrent lors de la Biométhanisation sont observables sur l'azote, surtout sa forme chimique : à l'issue de la Biométhanisation, l'azote sera principalement sous forme minérale (45-75\% d'ammonium) rapidement exploitable par les végétaux. Les 25-55 \% reliquats sont sous forme organique [27]. La tourbe, généralement usagée dans la fabrication des substrats horticoles, est un produit non renouvelable [28, 29]. Il serait intéressant de développer la recherche de produits organiques locaux alternatifs et d'encourager ainsi l'utilisation partielle des ressources de substitution, qui aident à conserver les avantages de la tourbe et permettent d'en limiter les quantités employées, et par conséquent, réduisant les importations et l'hémorragie locale des Devises. Dans cette perspective, l'investigation entreprise consiste à déterminer quelques critères de qualité physicochimique de trois Méthacomposts Avicoles (MCA) issus de la bioconversion anaérobie industrielle de la biomasse générée par un élevage intensif de poules pondeuses, en appréciant les possibilités de leur incorporation avec la tourbe, comme substrat de croissance des plants maraîchers.

\section{MATÉRIEL ET MÉTHODES}

\subsection{Site expérimental et digesteur mis en ouvre}

Il s'agit d'un digesteur industriel pilote de forme cylindrique, installé dans une ferme avicole à Hammam Sousse appartenant au gouvernorat de Sousse (Tunisie), à partir de l'année 2000, d'une capacité utile de $300 \mathrm{~m}^{3}$, alimenté en continu quotidiennement par $10 \mathrm{~m}^{3}$ de substrat formé 
d'environ $1 / 3$ de fientes avicoles et $2 / 3$ d'eau découlant lors du raclage des poulaillers avec l'effluent partant décanté (aboutissement aqueux ou surnageant). L'alimentation en biomasse avicole étant mécanique et le raclage des poulaillers admet une bonne dilution du substrat et un pompage facile [30].

Ce digesteur industriel cylindrique, disposé verticalement, est du type digesteur de contact anaérobie à cellules immobilisées (à biomasse fixée). Le garnissage est composé d'un support inerte de nature (6000 briques de 12). Un tel digesteur fonctionne avec un flux ascendant/descendant. Suivant ce procédé, l'affluent s'écoule vers le haut ou vers le bas (selon sa teneur en solides) à travers le digesteur contenant les briques de 12 qui retiennent les microorganismes anaérobies et sur lesquelles ils se multiplient.

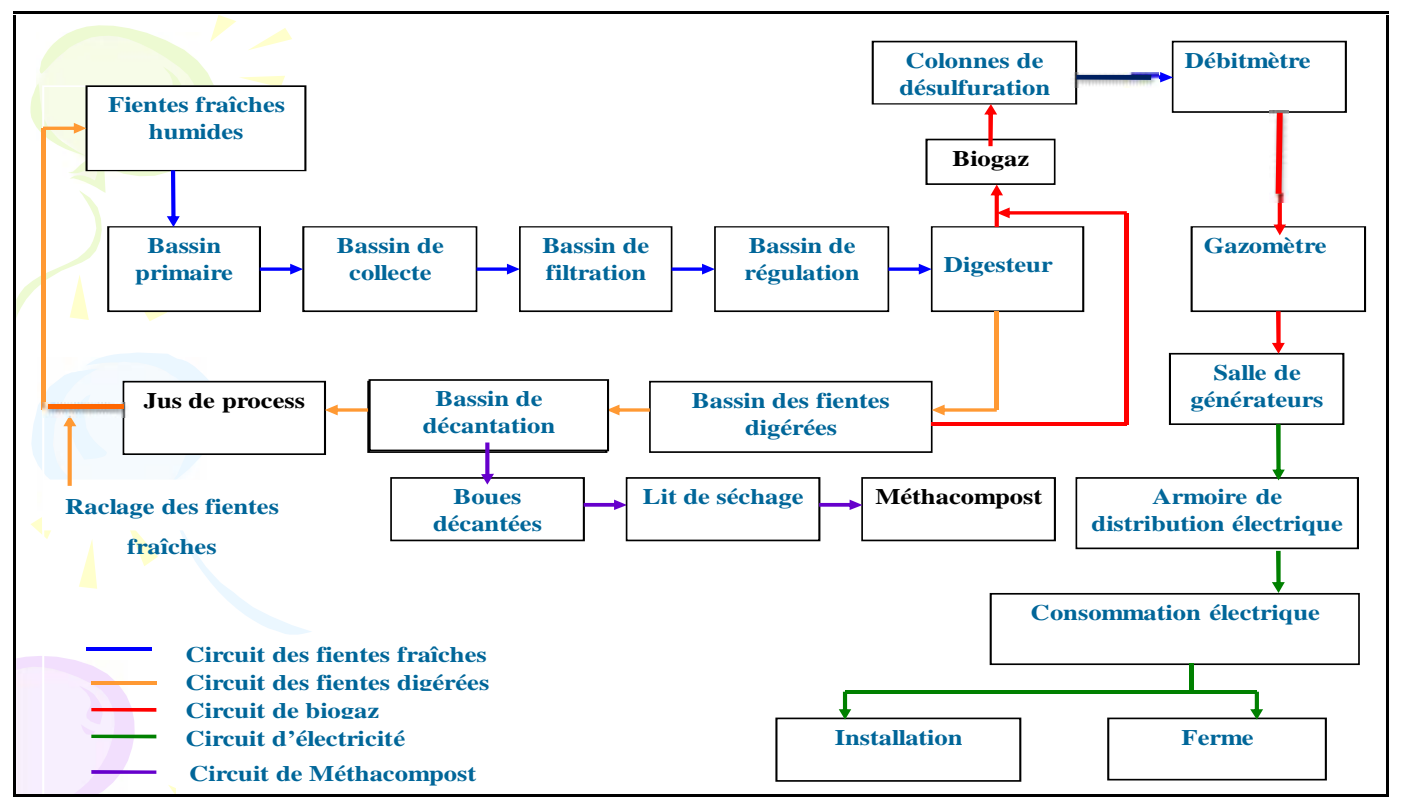

Fig.1. Schéma synoptique de divers circuits de la Biométhanisation industrielle avicole adoptée Les fientes digérées, engendrées dans le digesteur, franchissent trois bassins différents (Figure 1) : Un premier bassin recueillant le substrat digéré pour un faible temps de séjour (appelé bassin des fientes digérées) avant de demeurer dans le second bassin, appelé bassin de décantation ou décanteur. À partir de ce dernier, les boues des fientes décantées seront séchées et converties en méthacompost. Quant à l'eau surnageant (Jus de process) le bassin de décantation, elle passe dans le troisième bassin pour servir actuellement au raclage des fientes fraîches des poulaillers. 
Il convient de signaler que parallèlement à chaque opération d'alimentation par de nouvelles fientes, une même quantité de fientes traitées s'évacue à partir du trop plein vers le bassin de collecte des fientes digérées.

\subsection{Matériel expérimental}

Les MCA, sous-produits solides, objets de cette investigation, sont issus de la Biométhanisation industrielle pilote de la biomasse avicole, décrite précédemment.

Le biogaz avicole produit subissant une étape d'épuration (désulfuration avec l'hématite de fer) avant son emploi, le taux de $\mathrm{CH}_{4}$ a passé de 60 à $75 \%$ suite à l'épuration appliquée (soit, un rendement d'épuration de 25\%). Après épuration, il convient aussi de noter une nette amélioration du Pouvoir Calorifique Inférieur (PCI) qui a évolué de 5110 à $6389 \mathrm{kcal} / \mathrm{Nm}^{3}$ (soit également, un accroissement relatif de 25\%) [31].

Les trois MCA mis à l'étude sont relatés ci-après.

* $\mathrm{MCA}_{\text {dig }}$ : Méthacompost Avicole Affiné (Sortie Digesteur).

* MCA1 déc $:$ Méthacompost Avicole Affiné (Sortie Décanteur).

* MCA2 déc $:$ Méthacompost Avicole Affiné (Sortie Décanteur).

Les analyses physico-chimiques ont été faites durant la même année pour le $\mathrm{MCA}_{\text {dig }}$ et le $M C A 1_{\text {déc }}$, alors que celles relatives au $M C A 2_{\text {déc }}$ ont été réalisées lors d'une autre campagne de production pour apprécier les différences éventuelles conséquentes à l'état d'évolution.

\subsection{Caractérisation physique}

\subsubsection{Masse volumique apparente}

La masse volumique apparente (mva) n'est autre que la masse de l'unité de volume à l'état sec. La mesure de la mva a été accomplie strictement sur le MCA2déc. Il s'agit de mettre les échantillons dans l'étuve à une température de $105^{\circ} \mathrm{C}$ pendant 24 heures pour estimer la masse sèche [32]. Elle est exprimée par la formule ci-après.

$$
\mathrm{mva}\left(\mathrm{g} / \mathrm{cm}^{3}\right)=(\mathrm{Ms}-\mathrm{Mc}) / \mathrm{V}
$$

Ms : masse sèche de l'échantillon (g), Mc : masse de la capsule vide (g), V : Volume de la capsule de cc.

\subsubsection{Porosité et caractéristiques hydriques}

La porosité ou l'espace poral correspond à l'évaluation des espaces vides en rapport à l'encombrement total d'un substrat [33]. Pour $\mathrm{MCA}_{\text {dig }}$ et $M C A 1_{\text {déc }}$, l'appréciation de la porosité a été accomplie par le test standard de porosité. Le matériel utilisé pour ce test est formé 
notamment de: Conteneurs à 15 cavités, éprouvette graduée, clou de perçage, gobelets, chronomètre.

Les variables mesurées sont :

Volume total $(\mathrm{Vt})$ en $\mathrm{ml}=$ volume du conteneur relatif à 3 cavités du conteneur.

Volume d'eau versée $(\mathrm{Va})$ en $\mathrm{ml}=$ volume des pores : air et eau.

Volume d'eau recueillie $(\mathrm{Vr})$ en $\mathrm{ml}=$ volume des pores $:$ air.

Les paramètres étudiés sont :

Porosité totale : $\mathrm{Pt}(\%)=(\mathrm{Va} / \mathrm{Vt}) \times 100$

Porosité d'aération : $\mathrm{Pa}(\%)=(\mathrm{Vr} / \mathrm{Vt}) \times 100$

Porosité de rétention : $\mathrm{Pr}(\%)=\mathrm{Pt}-\mathrm{Pa}$

Pour l'autre méthacompost (MCA2 déc), l'estimation de la porosité a été accomplie en s'inspirant de la caractérisation hydrique. La détermination des propriétés des flux d'eau et d'air des substrats a été déduite en se basant sur les courbes $\mathrm{pF}$. La porosité totale $(\mathrm{Pt})$ est exprimée selon la relation entre le volume d'espaces vides $(\mathrm{Vv})$ et le volume total ou volume apparent (Va). Le volume apparent n'est autre que la somme du volume de la phase solide et du volume des vides. Ce paramètre est difficile à déterminer instantanément. Il en existe, en effet, plusieurs formulations qui diffèrent légèrement de l'une à l'autre [33], parmi lesquelles, on a eu recours à celle de Gras, utilisée par Mustin [34] et qui est exprimée par la formule ci-après.

$$
\operatorname{Pt}(\%)=95,83-32,43 \mathrm{mva}
$$

L'énergie avec laquelle l'eau est retenue par le milieu poreux va dépendre de l'aptitude du substrat, mais aussi de son humidité : on obtient une courbe caractéristique [35, 36] couramment appelée courbe de $\mathrm{pF}$ (potentiel of Free energy). L'établissement des courbes pF pour chaque type de substrat permet de les comparer et de les caractériser suivant leurs propriétés hydriques. Dans le domaine de la croissance végétative, les forces de succion exercées par les racines satisfont la hauteur d'eau comprise entre 0 et $20 \mathrm{~cm}$. De ce fait, le logarithme décimal de cette succion est adopté [33, 35, 37].

$$
\log 10=\mathrm{pF}
$$

Les échantillons des substrats ont été placés en contact avec une colonne d'eau en combinaison avec une couche de sable saturée d'eau (table à succion). On opère par dessiccation d'un produit qui a été au préalable amené à saturation complète et on soumet alors l'échantillon à une succion 
correspondant à la valeur choisie. Le potentiel hydrique de l'échantillon se met en équilibre avec la pression hydrostatique de la colonne d'eau.

Une telle technique est physiquement limitée à -100 mbar ( $\mathrm{pF}$ 2). Il s'agit d'un bac à sable utilisé pour l'estimation du $\mathrm{pF}$ de 0 à 2 . Les potentiels hydriques sont acquis en produisant une série de dépressions et surpressions. La pesée de chaque échantillon après adaptation exprime le chargement d'eau pour chaque tension. Le substrat testé est symbolisé par deux capsules dont chacune représente une répétition. Selon Gras [35], la teneur en air est déterminée après avoir établi la courbe pF. Elle est complémentaire de la teneur en eau, puisque ces deux fluides se partagent l'espace poral. On a donc la relation ci-après donnée par Morard [33]

$$
\mathrm{P}_{\mathrm{t}}(\% \mathrm{vol})=\text { Teneur en eau }(\% \mathrm{vol})+\text { Teneur en } \operatorname{air}(\% \text { vol })
$$

Avec :

Teneur en air à pF $1(\%$ vol $)=\mathrm{Pt}(\%$ vol $)-\mathrm{HV}$ à $\mathrm{pF} 1(\%)$

Teneur en air à $\mathrm{pF} 1$ = Porosité d'aération $(\mathrm{Pa})$

Teneur en eau à $\mathrm{pF} 1=$ Porosité de rétention $(\mathrm{Pr})$

L'Humidité Volumique (HV) à pF 1 a été déterminée en faisant appel à la formule ci-après.

$$
\mathrm{HV} \text { à pF } 1(\% \text { vol })=\frac{\text { Masse à pF1 }- \text { Masse sèche totale }}{\text { Volume de la capsule }}
$$

\subsection{Caractérisation chimique}

\subsection{1. $\mathrm{pH}$}

Le pH est déterminé après mise en solution de $20 \mathrm{~g}$ de l'échantillon, dans $100 \mathrm{ml}$ d'eau distillée. La méthode utilisée consiste à préparer une suspension du substrat dilué séché dans 5 fois son volume d'eau ( $1: 5)$, la laisser en agitation durant $5 \mathrm{mn}$, ensuite la faire reposer pendant au moins deux heures. La lecture du $\mathrm{pH}$ se fait par un pH-mètre.

\subsubsection{Conductivité électrique}

La Conductivité Électrique (CE) permet de déterminer la concentration en ions solubles d'un substrat par le biais de la propriété d'une solution aqueuse de conduire l'électricité relativement à sa concentration en ions [38]. Couramment exprimée en $\mathrm{mS} / \mathrm{cm}$ ou $\mathrm{mmhos} / \mathrm{cm}^{3}$, elle est mesurée à l'aide d'un conductimètre. Un échantillon de substrat est extrait avec de l'eau à $20 \pm 1{ }^{\circ} \mathrm{C}$ (Rapport d'extraction de 1:5 pour dissoudre les électrolytes). 


\subsubsection{Matière organique}

L'estimation de la Matière Organique (MO) et des cendres est opérée suivant la Norme Tunisienne (NT) relative au dosage de la MO du fumier. Pour apprécier le taux de MO de chaque substrat, il faut peser $20 \mathrm{~g}$ que l'on met dans l'étuve durant 24 heures à $70^{\circ} \mathrm{C}$, ensuite on fait la calcination de $3 \mathrm{~g}$ préalablement séchés pendant 2 heures à l'étuve, à $900^{\circ} \mathrm{C}$ durant au moins 6 heures dans un four à moufle et on détermine le Résidu Sec (RS).

La teneur en MO est déterminée, selon la formule suivante :

$$
(\%) \mathrm{MO}=[(\mathrm{M} 1-\mathrm{M} 2) / \mathrm{M} 1] \times 100
$$

Avec :

M1 : Masse avant calcination $(\mathrm{mg})$

$\mathrm{M} 2$ : Masse après calcination $(\mathrm{mg})$.

En partant de l'expression de la MO, exigée selon la Norme Tunisienne (NT), une déduction de la teneur en carbone est possible, en appliquant la relation ci-après.

$$
\begin{aligned}
& (\%) \mathrm{MO}=1,4 \times \mathrm{C}_{\mathrm{org}}(\%)+1,5(\mathrm{NT}) \\
& (\%) \mathrm{C}_{\mathrm{org}}=(\mathrm{MO}-1,5) / 1,4(\mathrm{NT})
\end{aligned}
$$

\subsubsection{Dosage de l'azote}

L'azote est dosé par la méthode de Kjeldhal [39] dont le principe s'appuie sur l'attaque de l'extrait par l'acide sulfurique concentré $\left(\mathrm{H}_{2} \mathrm{SO}_{4}\right)$.

\subsubsection{Dosage de certains Éléments minéraux}

Le dosage des éléments chimiques a touché aussi le phosphore (P) et le potassium (K). Il sollicite une minéralisation et la préparation des solutions d'étalonnage. La minéralisation étant une étape commune, seulement la nature et la solution d'étalonnage diffèrent d'un paramètre chimique à un autre. La détermination du pourcentage de chaque élément s'effectue comme suit.

$$
\mathrm{P}, \mathrm{K}(\%)=(\mathrm{n} \times \mathrm{D} \times \mathrm{V}) /\left(\mathrm{p} \times 10^{4}\right)
$$

Avec :

$\mathrm{n}$ : valeur trouvée $(\mathrm{g} / \mathrm{ml})$

$\mathrm{D}$ : dilution de la solution à photomètre $(\mathrm{D}=1)$

$\mathrm{V}$ : volume de la solution $(\mathrm{V}=100 \mathrm{ml})$

$\mathrm{p}$ : prise d'essai $(\mathrm{g})$ 
Les formes minérales sont les formes oxydées d'éléments majeurs. Elles sont acquises en se basant sur la calcination de la MS. Les $\%$ de $\mathrm{P}_{2} \mathrm{O}_{5}$ et de $\mathrm{K}_{2} \mathrm{O}$ ont été déterminés en faisant appel aux deux expressions suivantes :

$$
\begin{aligned}
& \mathrm{P}_{2} \mathrm{O}_{5}(\%)=2,3 \times \mathrm{P}(\%) \\
& \mathrm{K}_{2} \mathrm{O}(\%)=1,2 \times \mathrm{K}(\%)
\end{aligned}
$$

Pour des raisons matérielles, $\mathrm{P}$ et $\mathrm{P}_{2} \mathrm{O}_{5}$ ont été évalués uniquement pour $\mathrm{MCA}_{\text {dig }}$ et $M C A 1_{\text {déc }}$.

\section{RÉSULTATS ET DISCUSSION}

\subsection{Appréciation du comportement physique des substrats}

\subsubsection{Résultats physiques globaux}

Cette évaluation a concerné tant les paramètres physiques relatifs à la masse volumique apparente (mva) que ceux correspondants aux trois porosités caractérisant un substrat de croissance sur le plan physique, à savoir : Porosité totale $(\mathrm{Pt})$, d'aération $(\mathrm{Pa})$ et de rétention $(\mathrm{Pr})$. Les diverses variables physiques relevées pour chaque substrat considéré sont rassemblés dans le tableau 1. Rappelons que les résultats de porosité ont été obtenus selon deux méthodes différentes, ce qui dégage des normes retenues variées.

\begin{tabular}{|c|c|c|c|c|}
\hline MCA & Paramètres & $\begin{array}{c}\text { Résultats } \\
\text { moyens }\end{array}$ & Norme & Référence \\
\hline \multirow[t]{3}{*}{$\mathbf{M C A}_{\text {dig }}$} & $\mathrm{Pt}(\%)$ & 67,30 & $\mathrm{Pt} \quad \geq 50$ & \multirow{6}{*}[40,41,42]{} \\
\hline & $\mathrm{Pa}(\%)$ & 11,20 & $\mathrm{~Pa} \geq 20$ & \\
\hline & $\operatorname{Pr}(\%)$ & 56,10 & $\operatorname{Pr} \geq 30$ & \\
\hline \multirow[t]{3}{*}{ MCA1 déc } & $\mathrm{Pt}(\%)$ & 70,60 & $\mathrm{Pt} \geq 50$ & \\
\hline & $\mathrm{Pa}(\%)$ & 12,10 & $\mathrm{~Pa} \geq 20$ & \\
\hline & $\operatorname{Pr}(\%)$ & 58,50 & $\operatorname{Pr} \geq 30$ & \\
\hline \multirow[t]{4}{*}{ MCA2 déc } & $\mathrm{mva}\left(\mathrm{g} / \mathrm{cm}^{3}\right)$ & 0,78 & $0,10<\operatorname{mva}<0,30$ & {$[19,37]$} \\
\hline & $\mathrm{Pt}(\%)$ & 70,54 & $80<\mathrm{Pt}<95$ & \multirow{3}{*}[37,40,43]{} \\
\hline & $\mathrm{Pa}(\%)$ & 16,19 & 20 à 30 & \\
\hline & $\operatorname{Pr}(\%)$ & 54,35 & 55 à 70 & \\
\hline
\end{tabular}

Tableau 1. Qualité physique des méthacomposts avicoles étudiés

\subsection{2. Évaluation de la masse volumique apparente}


Le MCA2 Déc présente une mva très élevée $\left(0,78 \mathrm{~g} / \mathrm{cm}^{3}\right)$ dépassant amplement les valeurs habituelles relatives aux supports de culture : $0,10<\mathrm{mva}\left(\mathrm{g} / \mathrm{cm}^{3}\right)<0,30$, selon Morel et al [37]. Ce matériau est considéré comme substrat lourd.

Les constatations relevées par Lamhamedi et al [44] assurent que la mva a des conséquences directes sur la croissance et la morphologie des racines. Le MCA2 déc ne peut dans aucun cas être utilisé à l'état pur, car il peut poser des contraintes majeures lors de son incorporation partielle avec la tourbe. A ce propos, il faut rechercher son incorporation partielle, tout en optant vers la proportion de mélange adapté.

\subsection{3. Évaluation de la porosité}

La porosité du MCA peut être influencée, entre autres, par les dimensions des particules et la nature du substrat (rétenteur et/ou aérateur). Les caractéristiques tant qualité que composition du substrat de croissance réjouissent d'un rôle capital dans la production des plants de qualité. Un bon substrat de croissance possède une porosité totale de l'ordre de 80 à $90 \%$ comme la tourbe brune [43], mais elle peut atteindre 95\% [37, 44].

Les trois MCA possèdent des valeurs de Pt insuffisantes (Tableau 1) comparativement à la règle d'appréciation rapportée par divers auteurs [37, 40, 43] : $80<\mathrm{Pt}(\%)<95$. Cette situation peut être due aux dimensions granulométriques hétérogènes (affinage irrégulier). Ils présentent une Pr conforme à la valeur d'appréciation retenue et une Pa faible, quelque soit la norme appliquée. Les particules fines de tels substrats permettent de stocker plus d'eau dans les micropores et avoir ainsi une aptitude de rétention en eau (Pr) élevée, et par conséquent, ils peuvent être vus comme substrats rétenteurs.

En se basant sur les normes énoncées par divers auteurs [40, 41, 42], les deux MCA mis à l'essai de porosité (selon le test standard) montrent dans un tel contexte des porosités Pt et $\mathrm{Pr}$ convenables, mais la $\mathrm{Pa}$ reste toujours non conforme. Il convient de signaler que les conditions Tunisiennes exigent les proportions de porosité suivantes : $\mathrm{Pt} \geq 50 \%, \mathrm{~Pa} \geq 20 \%$ et $\mathrm{Pr} \geq 30 \%$. Ces règles à révérer ont été emportées des normes canadiennes [40] en encourageant la rétention sur l'aération, choix dicté par le climat sec de la Tunisie [41, 42].

Un bon substrat doit répondre à certains critères notamment une bonne aptitude de retenir l'eau, à drainer et à se réhumidifier facilement [43]. La tourbe est largement employée en pépinière comme un composant principal des substrats de croissance grâce à sa grande aptitude de rétention 
d'eau [44]. Les MCA présentent une capacité de rétention en eau proche de celle de la tourbe, alors que leur aptitude de rétention en air est loin d'être satisfaisante.

D'après Morard [33], on recherche actuellement à utiliser un substrat de bonnes Pt, Pa et Pr. D'où, les trois MCA étudiés, à l'état pur, devraient être évités pour ne pas affecter l'aération, en réduisant l'espace lacunaire favorisant ainsi l'empâtement du substrat. Il n'existe pas jusqu'à présent des matériaux réellement alternatifs à la tourbe de point de vue qualité physique. En plus, le MCA, dans les trois états considérés, exige un substrat aérateur, afin de corriger la $\mathrm{Pa}$ et obtenir un équilibre physique proprement dit. Il est nécessaire dans ce contexte, de rechercher le meilleur ratio de mélange pour une optimisation physique adéquate.

\subsection{Appréciation du comportement chimique des substrats}

\subsection{1. Évaluation du pH et de la conductivité électrique}

Le tableau 2 récapitule le $\mathrm{pH}$ et la Conductivité Électrique (CE) relevés pour chaque substrat étudié.

Tableau 2. pH et CE des méthacomposts avicoles considérés

\begin{tabular}{lll} 
& $\mathbf{p H}$ & $\mathbf{C E}(\mathbf{m S} / \mathbf{c m})$ \\
\hline Norme & $7<\mathrm{pH}<9$ & $0,5<\mathrm{CE}<2$ \\
\hline Référence & {$[45,46]$} & {$[37]$} \\
\hline MCA $_{\text {dig }}$ & 8,20 & 2,90 \\
\hline MCA1 $_{\text {déc }}$ & 8,70 & 2,60 \\
\hline MCA2 $_{\text {déc }}$ & 7,43 & 1,30
\end{tabular}

Le $\mathrm{pH}$ pourrait être un indice de la maturité complète d'un substrat. La valeur du $\mathrm{pH}$ d'un compost mûr se situe normalement entre 7 et 8 [45], voire entre 7 et 9 [46]. Les MCA testés peuvent être vus comme des substrats mûrs, étant donné qu'ils présentent des valeurs de $\mathrm{pH}$ répondant à la valeur normative retenue (Tableau 2).

La CE peut éclaircir la disponibilité des éléments minéraux dans le milieu de culture. La teneur élevée en sels affecte le comportement végétatif, pouvant ainsi déclencher des brûlures aux racines et aux feuillages. Il faut garder en mémoire que les plantes s'enracinent mieux dans un substrat contenant peu d'éléments nutritifs [47]. En effet, une faible valeur représente une petite quantité d'ions en solution, ce qui rend plus rude l'absorption d'eau et d'éléments nutritifs par les végétaux et peut même brûler les racines. La minéralisation d'azote et la production d'acides organiques sont généralement à l'origine de l'amplification de la salinité [38]. La 
Biométhanisation, au contraire, réduit davantage la salinité du matériau de départ (fientes avicoles) du fait que les excréments d'animaux sont reconnus par leur teneur élevée en sels. Selon Fuchs et al [48], la CE du compost ne doit pas dépasser $4 \mathrm{mS} / \mathrm{cm}$. La salinité excessive d'un compost peut être la source de sa phytotoxicité [38].

En se rapportant aux constatations rapportées par C.P.V.Q. [40] et Comtois et Legaré [47], des carences en $(\mathrm{Fe}, \mathrm{Cu}, \mathrm{Mn}, \mathrm{B})$ peuvent se produire et des excès possibles en $(\mathrm{Ca}, \mathrm{N})$ peuvent apparaître à l'échelle du substrat. Soumaré et al [49] affirment que les substrats de croissance devraient avoir une faible $\mathrm{CE}$ inférieure à $3 \mathrm{mS} / \mathrm{cm}$. Au-delà de cette valeur, des suites négatives pourraient avoir lieu sur la germination et l'émergence des semences. Selon Morel et al [37], la CE est acceptable entre 0,5 et $2,0 \mathrm{mS} / \mathrm{cm}$. En se basant sur cette dernière norme, le MCA2 déc présente une valeur conforme $(1,30 \mathrm{mS} / \mathrm{cm})$, alors que les deux autres renferment des valeurs élevées (Tableau 2).

\subsubsection{Détermination de la fraction organique}

Le tableau 3 résume la $\mathrm{MO}$, l'azote et le rapport $\mathrm{C} / \mathrm{N}$ relevés pour chaque substrat considéré

Tableau 3. \% MO, \% $\mathrm{N}$ et $\mathrm{C} / \mathrm{N}$ des méthacomposts avicoles étudiés

\begin{tabular}{llll} 
& $\mathbf{M O}(\boldsymbol{\%})$ & $\mathbf{N}(\boldsymbol{\%})$ & $\mathbf{C} / \mathbf{N}$ \\
\hline Norme & $35<\mathrm{MO}<45$ & $0,92<\mathrm{N}<2,76$ & $8<\mathrm{C} / \mathrm{N}<15$ \\
\hline Référence & {$[43]$} & {$[48]$} & {$[35]$} \\
\hline MCA $_{\text {dig }}$ & 32,50 & 2,40 & 7,50 \\
\hline MCA1 $_{\text {déc }}$ & 36,50 & 2,60 & 7,80 \\
\hline MCA2 $_{\text {déc }}$ & 37,33 & 2,28 & 11,25
\end{tabular}

Le taux de MO est d'une importance fondamentale pour la valeur fertilisante du substrat, du fait de ses effets physico-chimiques et biologiques. La tourbe est largement employée en pépinière comme un composant principal des substrats de croissance grâce à sa haute teneur en MO [43] D'après Levasseur et Aubert [50], les fientes des poules pondeuses présentent un taux de MO de l'ordre de $58 \%$.

La Biométhanisation est la source d'une réduction significative du taux de MO. La dégradation importante de la MO des déjections générées par les poules pondeuses pendant la Digestion Anaérobie (DA) est expliquée par les résultats trouvés pour les trois méthacomposts étudiés qui sont respectivement de l'ordre de 32,50\%,36,50\% et 37,33\% pour MCAdig, MCA1déc et 
MCA2déc (Tableau 3). Un degré de dégradation trop élevé des fientes avicoles, résultant d'une durée de digestion plus longue, risque de diminuer la valeur organique du MCA produit. Ce résultat est conforme avec celui dégagé par ADEME [51] qui a annoncé que le digestat produit est pauvre en MO.

D'après les directives rapportées par Fuchs et al [32], le compost mûr doit avoir une teneur en MO inférieure à 50\% (\% MS). Pour un compost de qualité, notamment maturité et stabilité, le taux de MO devrait être compris entre 35 et $45 \%$ (\% MS). Selon ce critère, les MCA sont considérés comme des produits mûrs et stables.

\subsection{3. Évaluation du rapport $\mathrm{C} / \mathrm{N}$}

La DA est la source d'une réduction significative du taux de MO. Malgré cette forte diminution, les teneurs en MO des MCA sont encore élevées pour permettre la confection d'un substrat de bonne qualité. La minéralisation de l'azote est importante, lors de la DA. Elle est proportionnelle au taux de biodégradation du carbone [52].

Les teneurs en azote des MCA (Tableau 3) sont adéquates conformément aux normes décrites par Vanai [43].

Selon ces dernières, un compost destiné à être utilisé en horticulture, doit avoir un taux d'azote supérieur à $1 \%$ de MS. La valeur minimale imposée par la directive de la Communauté Européenne est de l'ordre de $0,6 \%$ alors que la valeur maximale imposée par la Norme Française NF U 44-051 est d'environ $2 \%$ pour les supports de culture. Au delà d'une valeur de $2 \%$, le compost devrait être vu comme un engrais organique [43]. La teneur relevée dépend de l'indice de maturité qui est souvent apprécié par le rapport C/N. Selon Fuchs et al [48], la teneur d'azote doit être comprise entre 0,92 et $2,76 \%$. Les teneurs élevées en azote des MCA sont dues à la richesse en azote des déjections animales en général, et des fientes avicoles, en particulier.

Étant donné que les MCA possèdent des teneurs assez élevées qui dépassent les teneurs acceptables relatives aux supports de culture, ces matériaux organiques ne peuvent pas être employés uniques comme supports de culture. Ils conduisent généralement à un affaiblissement des endurances mécaniques des végétaux et à une importante sensibilité à certaines maladies cryptogamiques [53].

Le rapport $\mathrm{C} / \mathrm{N}$ est fréquemment adopté pour apprécier le processus de minéralisation de la biomasse organique [54] et comme indicateur de maturité des composts [45]. Selon Vanai [43], ce rapport varie largement selon l'origine des déchets compostés et il est influencé directement 
par la teneur relative en azote du substrat, alors que le rapport $\mathrm{C} / \mathrm{N}$ d'un compost est insuffisant pour évaluer sa maturité [55]. Les faibles rapports $\mathrm{C} / \mathrm{N}$ relevés des MCA peuvent s'expliquer par le fait que la minéralisation des composts d'origine animale est plus rapide que celle d'origine végétale (dégradation difficile des polymères).

Selon Lemaire et al [56], les MO avec rapport $\mathrm{C} / \mathrm{N}$ faible ou moyen ne conviennent pas pour l'obtention de supports de culture, car elles évoluent temporellement par minéralisation. Il en résulte des tassements, des variations de porosité liées aux pertes en MS et au colmatage par les éléments fins ou colloïdaux. Une concurrence pour l'oxygène entre les microorganismes apparaît, plus nettement lorsque la porosité diminue.

Le $\mathrm{C} / \mathrm{N}$ décroit constamment pendant le compostage pour se stabiliser vers 10 (8 à 15) dans un compost mûr [34]. Ce paramètre présente néanmoins un défaut majeur, à cause de l'absence de valeurs de référence précises. Partant de la norme annoncée, on peut exprimer que le $\mathrm{C} / \mathrm{N}$ est acceptable dans les trois cas (Tableau 3). Jiménez et Garcia, cités par Larbi [45] suggèrent un rapport inférieur à 12 qui reflète la bonne maturité du compost. Brasseur et Sullivan, cités par Tambone et al [57] ont proposé un $\mathrm{C} / \mathrm{N}$ idéal s'étendant de 12 à 25 . Cette dernière norme pénalise les résultats trouvés. La valeur normative de 8 à 15 est généralement admise.

\subsection{4. Évaluation des taux de phosphore et de potassium}

Le tableau 4 regroupe les divers résultats relatifs aux teneurs en éléments minéraux ( $\mathrm{P}$ et $\mathrm{K}$ ) analysés pour chaque substrat étudié.

Tableau 4. Éléments minéraux relevés pour les méthacomposts avicoles considérés

\begin{tabular}{lcccc} 
& $\mathbf{P}(\boldsymbol{\%})$ & $\mathbf{P}_{\mathbf{2}} \mathbf{O}_{\mathbf{5}}(\boldsymbol{\%})$ & $\mathbf{K}(\boldsymbol{\%})$ & $\mathbf{K}_{\mathbf{2}} \mathbf{O}(\boldsymbol{\%})$ \\
\hline Norme & 0,7 à 0,9 & 1,145 à 2,061 & $\mathrm{~K}>0,25$ & $\mathrm{~K}_{2} \mathrm{O}>0,3$ \\
& $(\% \mathrm{MS})$ & $(\% \mathrm{MS})$ & & \\
\hline Référence & {$[41]$} & {$[41]$} & {$[41]$} & {$[41]$} \\
\hline MCA $_{\text {dig }}$ & 0,75 & 1,72 & 1,73 & 2,08 \\
\hline MCA1 déc & 0,78 & 1,79 & 1,86 & 2,23 \\
\hline MCA2 déc & - & - & 1,83 & 0,30
\end{tabular}

Généralement, le taux de $\mathrm{P}$ dans le compost varie de 0,7 à $0,9(\% \mathrm{MS})$ dont 50 à $60 \%$ sont assimilables. Les valeurs relevées pour les deux MCA mis à l'essai répondent à cette fourchette (Tableau 4). Le MCA est un matériau riche en K. Les teneurs moyennes en K des MCA produits 
par Biométhanisation sont de l'ordre de 1,73\%, 1,86\% et 1,83\% successivement pour les trois MCA (Tableau 4). Les teneurs en $\mathrm{K}_{2} \mathrm{O}$ sont hautement supérieures à la valeur minimale imposée par la Directive de la Communauté Européenne $\left(0,3 \%\right.$ en $\left.\mathrm{K}_{2} \mathrm{O}\right)$, cité par [41] pour admettre la vente des composts. La richesse globale en éléments minéraux des MCA pourrait constituer un bon indice pour réduire le nombre des fertigations pratiquées en pépinière, d'où le double intérêt des MCA comme substrats rétenteurs d'eau (rôle physique) et riches en éléments minéraux. Par contre, leur aération est insuffisante.

\section{CONCLUSION}

La valorisation agronomique des résidus ou digestats solides de la Biométhanisation avicole s'avère intéressante, tout en réclamant une qualité et une efficacité maximale pour une meilleure exploitation en pépinière hors sol.

Les digestats solides ou Méthacomposts Avicoles (MCA), objet de cette étude, ont une granulométrie fine des particules, pouvant créer des barrières aux échanges gazeux (manque d'aération et excès de rétention d'eau). Tenant compte de cette contrainte physique majeure et de la masse volumique apparente importante, ils peuvent être utilisables uniquement comme des substituts partiels de la tourbe dans la fabrication des substrats de culture. Leur incorporation à raison de 10 à $20 \%$ pourrait être une alternative possible et rentable.

Concernant la qualité chimique, les MCA ont montré des $\mathrm{pH}$ et des salinités relativement acceptables. Donc, ils peuvent être assurés mûrs et corrélativement stables et peuvent être employés en système hors sol avec énormément de précautions. Les MCA étudiés présentent un taux de MO insuffisant, mais ils sont riches en azote, tout en enregistrant des taux de phosphore et de potassium relativement valables.

Dans l'ensemble, les résultats acquis au cours de cette étude indiquent des effets non négligeables des méthacomposts avicoles sur plusieurs paramètres physiques, hydriques et chimiques des substrats de croissance confectionnés. Cette constatation justifie leur emploi uniquement comme substitut partiel de la tourbe permettant ainsi de réduire en partie les quantités importées.

En définitive, il convient de poursuivre les études en vue de caractériser parfaitement les digestats et d'évaluer leur capacité à être usagés en valorisation agronomique directe ou à être transformés via des post-traitements ultérieurs. 


\section{RÉFÉRENCES}

[1] Oost J.Fr., De Toffoli M. Estimation de la valeur fertilisante de digestats issus de la biométhanisation, Centre Pilote de Maïs, Belgique, 2011, 5 p.

[2] Goyal H.B., Seal D. and Saxena R.C. Renewable and sustainble energy reviews, 12 (2), 2008, 507-517.

[3] Bridgwater A.V. Chemical Engineering Journal, vol. 91, 2003, 87-102.

[4] Yokoyama S. Jonouchi K. and Imou K. Processings of wolds academy of science, engineering and technology, vol. 22, 2007, 320-323.

[5] Demirbas A. Energy conversion and management, vol. 42, 2001, 1357-1378.

[6] Gijzen H. Water Science and Technology, 45 (10), 2002, 321-328.

[7] Hess J. Modélisation de la qualité du biogaz produit par un fermenteur méthanogène et stratégie de régulation en vue de sa valorisation. Thèse de Doctorat, Université de Nice- Sophia Antipolis-UFR Sciences, 2007, 228 p.

[8] Afilal M.E. Bakx A. Belakhdar N. and Membrez Y. Rev. Energ. Ren., 13 (2), 2010, 249-255.

[9] Essabri E. et El Omari H. Rev. Microbiol. Ind. San. et Environn., 6 (2), 2012, 164-173..

[10] M'Sadak Y. and Ben M'Barek A. J. Fundment. Appl. Sci., 6 (1), 2014, 11-30.

[11] Afilal M.E., Elasri O. et Merzak Z. J. Mater. Environ. Sci., 5 (4), 2014, 1160-1169.

[12] M'Sadak Y. and Ben M'Barek A. J. Fundment. Appl. Sci., 7 (2), 2015, 185-202.

[13] Afilal M.E., Moncif M. et Benyamna A. Rev. Energ. Ren. CER'07 Oujda , 2007, 9-12.

[14] M'Sadak Y., Ben M'barek A., Zoghlami R.I. et Baraket S. Rev. Energ. Ren., 14 (2), 2011, 343-356.

[15] M'Sadak Y. et Zoghlami R.I. Alg. J. of Ar. Env., 2 (2), 2012, 16-27.

[16] M'Sadak Y., Ben M'Barek A. et Zoghlami R.I. Revue Nature \& Technologie, $N^{\circ} 8$ (C), 2013, 19-26.

[17] M'Sadak Y. and Ben M'Barek A. International Journal of Innovation and Applied Studies, 4 (2), 2013, 343-352.

[18] Afilal M.E., Belkhadir N. and Merzak Z. Global Journal of Science Frontier Research Biological Sciences, Vol. XIII Issue I, 2013, 3 p.

[19] Afilal M.E., Elasri O. et Merzak Z. J. Mater. Environ. Sci. 5 (4), 2014, 1160-1169.

[20] Chouari R., Guermazi S., Riviere D., Pelletier E., Weissenbach J., Le Paslier D. et Sghir A. Actes Journées Industrielles Méthanisation, 2012, 18-28. 
[21] Tou I., Igoud S. et Touzi A. Rev. Energ. Ren. : Production et Valorisation-Biomasse, 2001, 103-108.

[22] M'Sadak Y. et Ben M'Barek A. Rev. Energ. Ren. 16 (1), 2013, 33-42.

[23] M'Sadak Y., Ben M'Barek A., Zoghlami R.I. et Baraket S. Revue de Génie Industriel, 7, 2012, 46-55.

[24] Martel S. et Desmeules X. Recherche et Innovation en Agriculture (AGRINOVA), Canada, 2013, 23 p.

[25] Martel S., Desmeules X., Landry Ch., Lavallée S., Paré M. et Tremblay F. Recherche et Innovation en Agriculture (AGRINOVA), Canada, 2013, 3 p.

[26] A.D.E.M.E. et Chambre d'Agriculture du Doubs. Fiche $\mathrm{N}^{\circ} 3$, Intérêt agronomique du digestat issu de la méthanisation, France, Mars 2006.

[27] Muller F. Rapport Final, Étude réalisée pour le compte de l'ADEME et le Ministère de l'Agriculture par RITTMO Agroenvironnement, Uteam, FIBL, INERIS, LDAR, France, Octobre 2011, $250 \mathrm{p}$.

[28] Gauthier F., Gagnon S. et Dansereau B. Canadian Journal of Plant Science, 1998, vol. 78, 131-138.

[29] Van Der Gaag D.J., Van Noort F.R. and Stapel-Cuijpers L.H.M., Kreij C., Termorshuizen A.J., Van Rijn E., Zmora-Nahum S. and Chen Y. Scientia Horticulturae. 2007, 114 (4), 289-297.

[30] ALCOR, AXENNE. Rapport final de l'Agence Nationale des Énergies Renouvelables (A.N.E.R.), 2003, 148-157.

[31] M'Sadak Y., Ben M'Barek A. et Zoghlami R.I. Revue Nature \& Technologie, 5, 2011, 5765.

[32] A.O.A.C., 'Official methods of analysis', Association of official analytical chemist (aoac), Washington, DC, 16ème Edition, 1990.

[33] Morard P. Les Cultures Végétales Hors Sol, Publications Agricoles Agropôle d'Agen, 1995, $304 \mathrm{p}$.

[34] Mustin M. Le Compost : Gestion de la Matière Organique, Edition François Dubusc, 1987, $954 \mathrm{p}$.

[35] Gras R. Revue Sc. Sol, 1981, 171-186.

[36] Gras R. et Aigus I. PHM Revue Horticole, 1982, 51-53. 
[37] Morel P., Poncet P. et Rivière L.M. Les supports de culture horticoles : Les matériaux complémentaires et alternatifs à la tourbe, Éditions INRA, 2000, 84 p.

[38] Tiquia S.M. Chemosphere, 79 (5), 2010, 506-512,.

[39] Goyal S., Dhull S.K. and Kapoor K.K. Bioresource Technology, vol. 96, 2005, 1584-1591.

[40] C.P.V.Q. Pépinières, Culture en conteneurs, Substrats, Document technique réalisé par le Conseil des Productions Végétales du Québec (C.P.V.Q.), 1993, 19 p.

[41] M'Sadak Y., Ben M’Barek A. et Tayachi L. Revue Nature \& Technologie, Nº, 2012, 59-70.

[42] M'Sadak Y., Elouaer M.A. et Dhahri M. Revue Nature \& Technologie, Nº9B, 2013, 27-34.

[43] Gras R. et Agius I. PHM Revue Horticole, vol. 234, 1983, 11-13.

[44] Lamhamedi M.S., Fortinn J.A., Ammari Y., Ben Jalloun S., Poirier M., Fecteau B., Bougacha A. et Godin L. Rapport technique : Exécution des Travaux d'Aménagement de Trois Pépinières Pilotes en Tunisie, 1997, 121 p.

[45] Larbi M. Influence de la Qualité des Composts et de leurs Extraits sur la Protection des Plantes contre les Maladies Fongiques, Thèse de Doctorat, Université de Neuchâtel, 2006, 161 p.

[46] Bernal M.P., Alburquerque J.A. and Moral R. Bioresource Technology, 100 (22), 2009, 5444-5453.

[47] Comtois M. et Legaré M. La Fertilisation des Plantes Ligneuses Cultivées en Contenant, Institut Québécois de Développement de l'Horticulture Ornementale, 2004, 57 p.

[48] Fuchs J.G., Galli U., Schleiss K. et Wellinger A. Association Suisse des Installations de Compostage (ASIC) \& Forum Biogaz Suisse, 2001, 26 p.

[49] Soumaré M., Demeyer A., Tack F.M.G. and Verloo M.G. Bioresource Technology, 81 (2), 2002, 97-101.

[50] Levasseur P. et Aubert C. Contexte, atouts et faiblesses des effluents porcins et avicoles destinés à être exportés, TECHNI-Porc, ESA d'Angers, 29 (2), 2006, 9 p.

[51] A.D.E.M.E. Gestion des déchets organiques en France. Le point sur les filières de gestion biologique avec retour au sol, 2006, $22 \mathrm{p}$.

[52] Pouech P. Journée Technique Nationale, 'Réussir un projet de méthanisation associant des déchets ménagers agricoles et industriels', 2008, 6 p.

[53] Ameziane El Hassani T. et Persoons E. Agronomie moderne : Bases physiologiques et agrnomiques de la production végétale. Édition Hatier-AUPELF. UREF, 1994, 544 p. 
[54] Annabi M. Stabilisation de la structure d'un sol limoneux par des apports de composts d'origine urbaine : Relation avec les caractéristiques de leur matière organique, Thèse de Doctorat de l'Institut National Agronomique (INA) Paris-Grignon, 2005, 280 p.

[55] Serra-Wittling C., Houot S. and Barriuso E. Biology and Fertility of Soils, 20 (4), 1995, 226236.

[56] Lemaire F., Dartigues A., Rivière L.M. et Charpentier S. Cultures en pots et conteneurs : Principes agronomiques et applications, INRA, PHM, Revue Horticole, 1989, 184 p.

[57] Tambone F., Genevini P., D’Imporzano G. and Adani F. Bioresource. Technology, vol.100, 2009, 3140-3142.

How to cite this article:

M'Sadak Y. and Ben M'Barek A. Characterization qualitative and potentialities of utilization of methacomposts of poultry in the nurseries aboveground. J. Fundam. Appl. Sci., 2016, 8 (3), 875-893. 